\title{
OPEN The risk and protective factors of heightened prenatal anxiety and depression during the COVID-19 lockdown
}

\author{
Stefania Vacaru ${ }^{1 凶}$, Roseriet Beijers ${ }^{1,2}$, Pamela D. Browne ${ }^{1}$, Mariëlle Cloin ${ }^{3,4}$, \\ Hedwig van Bakel ${ }^{3,4}$, Marion I. van den Heuvel ${ }^{3}$ \& Carolina de Weerth ${ }^{1}$
}

While pregnant women are already at-risk for developing symptoms of anxiety and depression, this is heightened during the COVID-19 pandemic. We compared anxiety and depression symptoms, as indicators of psychological distress, before and during COVID-19, and investigated the role of partner, social network and healthcare support on COVID-19-related worries and consequently on psychological distress. A national survey, conducted during the first lockdown in The Netherlands, assessed COVID-19 experiences and psychological distress $(\mathrm{N}=1421)$, whereas a comparison sample $(\mathrm{N}=1439)$ was screened for psychological distress in 2017-2018. During COVID-19, the percentage of mothers scoring above the questionnaires' clinical cut-offs doubled for depression (6\% and $12 \%)$ and anxiety (24\% and 52\%). Women reported increased partner support during COVID-19, compared to pre-pandemic, but decreased social and healthcare support. Higher support resulted in lower COVID19-related worries, which in turn contributed to less psychological distress. Results suggest that a global pandemic exerts a heavy toll on pregnant women's mental health. Psychological distress was substantially higher during the pandemic than the pre-pandemic years. We identified a protective role of partner, social, and healthcare support, with important implications for the current and future crisis management. Whether increased psychological distress is transient or persistent, and whether and how it affects the future generation remains to be determined.

Pregnancy involves profound physiological and psychological changes in women ${ }^{1-3}$, due to the hormonal and biological changes in the body ${ }^{4,5}$, and due to the surrounding context ${ }^{6,7}$. These changes have been associated to elevated prenatal symptoms of anxiety and depression ${ }^{8-10}$, which in turn are related to poor outcomes in the offspring ${ }^{11-13}$. Accordingly, pregnant women's known vulnerability for anxiety and depression has become a concern during the COVID-19 pandemic and the severe lockdown measures, including social distancing, workrelated changes, and limited access to healthcare services ${ }^{14}$. Whilst reports show a general rise in anxiety and depression rates associated with COVID-19-related worries ${ }^{15-18}$, pregnant women might experience even more worries. These may include the uncertainty around the transmission of the virus in utero and its effects on the fetus, and the implications for healthcare and birth arrangements ${ }^{16,18-20}$. A recent meta-analysis found heightened mental health problems with $43 \%$ of pregnant women experiencing anxiety and $32 \%$ depression during the COVID-19 pandemic ${ }^{21}$. Yet, how and which risk or protective factors contribute to anxiety and depressive symptoms, henceforth 'psychological distress', during the COVID-19 pandemic remains unexplored.

Subjective experiences due to the COVID-19 pandemic may vary considerably across pregnant women. Availability of social support may play a significant role in managing COVID-19-related worries, contributing in turn to diminishing the risk of developing psychological distress ${ }^{22}$. According to the buffering hypothesis ${ }^{23}$, support from others, defined as regular positive interactions and integration in a larger network that provides help during hardship (financial, legal, health, emotional, etc.), is one of the most important protective factors from adverse events. Likewise, social support is a central focus of mental health interventions, such as in interpersonal psychotherapy that views psychiatric disorders as a precipitation of the interpersonal context and support systems ${ }^{24,25}$. In the context of physical distancing regulations, support systems are fundamental for

${ }^{1}$ Donders Institute for Brain, Radboud University Medical Center, Cognition \& Behavior, Nijmegen, The Netherlands. ${ }^{2}$ Radboud University, Nijmegen, The Netherlands. ${ }^{3}$ Tilburg University, Tilburg, The Netherlands. ${ }^{4}$ Tranzo Tilburg University, Tilburg, The Netherlands. ${ }^{\circledR}$ email: stefania.vacaru@radboudumc.nl 
pregnant women's mental health ${ }^{26}$. Three main support sources during the prenatal period are partner ${ }^{27}$, social network $^{28}$, and healthcare support ${ }^{29}$. Being housebound, it is likely that most of the social interactions take place within the couple or family that occupy a primary role in providing pregnant women with a safe psychological environment ${ }^{30,31}$, and in turn reduce the likelihood of psychological distress. Additionally, despite socializing dynamics having changed in line with governments' restrictions, the role of family and friends' support is more important than ever ${ }^{32}$. Pre-COVID-19 pregnancy studies showed that social support represents an important buffer against psychological distress ${ }^{33,34}$, thus begging the question whether social support might help decrease COVID-19 worries, reducing the risk for psychological distress. Likewise, access to healthcare services changed, with appointments being cancelled, postponed, and/or taking place virtually or telephonically. A recent study in the UK reported significant barriers for pregnant women in seeking healthcare during the pandemic, including lack of support, virtual care, and information communication ${ }^{35}$. And together, these drastic changes may result in heightened psychological distress.

This study had two main goals. Firstly, we aimed to replicate previous reports on prenatal mental health worsening during COVID-19 $9^{36,37}$, by comparing data from two Dutch cohorts, one assessed prior to and one assessed during the COVID-19 pandemic. Our second goal was to assess whether partner, social network, and healthcare support protected directly and indirectly against psychological distress. Accordingly, for the direct route, we investigated whether these support systems negatively predicted symptoms of anxiety and depression. For the indirect route, we investigated whether these support systems could lead to lower symptoms of anxiety and depression by specifically decreasing worries related to the COVID-19 pandemic.

\section{Methods}

Participants. COVID-19. This sample was recruited via an online questionnaire that was set up as part of a larger ongoing international multisite project (https://www.covgen.org/), during the most stringent lockdown restrictions (4th April-10th May 2020). The inclusion criterion was pregnant women when completing the survey. Two participants were excluded due to a pattern of random responses; hence, the final sample consisted of 1419 women. No other exclusion criteria was used. From the final 1419 participants, 747 had some missing data. These were generally pairwise-deleted ${ }^{21}$; yet for the mediation analyses, listwise deletion was performed ${ }^{38}$. Recruitment was performed through midwifery centers (14\%), word of mouth (15\%), social media (60\%), and others (11\%). The Ethics Review Board of Tilburg University [RP2019-143] approved the study, which was conducted according to the Declaration of Helsinki. Participants provided informed consent before completing the survey and were compensated with $10 €$.

Pre-COVID-19. A sample of 1439 Dutch pregnant women, recruited from March 2017-September 2018, constituted our comparison group. The inclusion criterion was pregnant women when completing the survey. This sample was recruited as part of an online questionnaire study investigating prenatal mental health treatment uptake $e^{8}$ and screening for participants for an intervention study ${ }^{39}$. An additional number of women were recruited but excluded from the study for not signing the informed consent $(\mathrm{N}=18)$, not completing the questionnaire $(\mathrm{N}=493)$ and for complicated pregnancies needing specialized treatment $(\mathrm{N}=36)$. Ninety-five percent of women were recruited via 2 ultrasound scan centers, while the rest via midwifery centers $(\mathrm{N}=21)$, a lactation practice $(\mathrm{N}=11)$, and social media $(\mathrm{N}=46)$. Women received an invitation email and upon providing informed consent, completed an online questionnaire. Ethical approval was obtained from the Faculty of Social Sciences of the Radboud University [ECSW2016-1710-42].

COVID-19 lockdown in The Netherlands. The Dutch lockdown in April/May 2020 entailed the closing of schools, day-care centers, restaurants, bars, gyms and other contact professions such as hairdressers, while shops were allowed to stay open, provided clients kept at a safe interpersonal distance ( $1.5 \mathrm{~m}$ rule). No curfew was implemented. Moreover, people were encouraged to work from home as much as possible and limit outings and social visits. Healthcare checkup visits, for example to a midwife, were unaccompanied and shortened, or delivered online or via telephone.

\section{Instruments}

Psychological distress. State-Trait Anxiety Inventory $(\mathrm{STAI})^{40}$ was used to assess anxiety symptoms. Here, we focused only on the state dimension, assessing anxiety feelings in the present with 20 items on a 4-point Likert scale ${ }^{41}$. Total scores vary between $20-80$, with scores $\geq 40$ indicating clinically relevant symptoms of anxiety ${ }^{42}$. Internal consistency was excellent in the pre-COVID (Cronbach's $\alpha=0.97$ ) and the COVID (Cronbach's $\alpha=0.94$ ) samples, as previously reported ${ }^{43}$.

Edinburgh Depression Scale (EDS) ${ }^{44}$ was used to assess depressive symptoms. This is a 10-item self-report questionnaire, on a 4 -point Likert scale ${ }^{45}$. Total scores range between $0-30$, with scores $\geq 13$ indicating clinically relevant depressive symptoms during pregnancy ${ }^{46}$. Consistent with previous evidence ${ }^{47}$, internal consistency was sound in the pre-COVID (Cronbach's $\alpha=0.88$ ) and the COVID sample (Cronbach's $\alpha=0.86$ ).

COVID-19 experiences. COVID-19 and Perinatal Experiences (COPE) ${ }^{48}$ was used to assess COVID-19-related experiences. This questionnaire is a comprehensive assessment of the impact of COVID-19 on one's life; COVID-19 exposure and symptoms, healthcare, finances, social support, community, coping, emotions, mental and physical health, addictions, and demographics. Here, we focused on (changes in) perceived support during the COVID-19 crisis and related stress. 


\begin{tabular}{|c|c|c|c|}
\hline & Pre-COVID sample $(N=1439)$ & COVID sample $(N=1419)$ & \multirow[b]{2}{*}{ Test statistic } \\
\hline & $M(S D, N)$ or $N(\%)$ & $M(S D, N)$ or $N(\%)$ & \\
\hline Age in years & $31.02(4.35,1418)$ & $30.77(3.84,976)$ & $t(2392)=1.42, p=.154$ \\
\hline \multicolumn{4}{|l|}{ Education } \\
\hline low & $90(6.3 \%)$ & $27(2.7 \%)$ & \multirow{3}{*}{$X^{2}=37.27, p<.001$} \\
\hline medium & $469(32.6 \%)$ & $254(25.3 \%)$ & \\
\hline high & $879(61.1 \%)$ & $724(72 \%)$ & \\
\hline \multicolumn{4}{|c|}{ Annual household income ${ }^{\star}$} \\
\hline$<€ 40,000$ & - & $235(23.5 \%)$ & \\
\hline$€ 40,000$ to $€ 100,000$ & - & $540(54.4 \%)$ & \\
\hline$>€ 100,000$ & - & $117(11.7 \%)$ & \\
\hline Parity (nulliparous) & $1351(48 \%)$ & $1419(48 \%)$ & $X^{2}=.01, p=.923$ \\
\hline Marital status (partner) & $1424(99 \%)$ & $979(97 \%)$ & $X^{2}=3.84, p=.050$ \\
\hline Gestation in weeks & $23.45(3.04,1439)$ & $24.98(9.44,1078)$ & $t(2515)=-5.77, p<.001$ \\
\hline \multicolumn{4}{|l|}{ Gestation trimester } \\
\hline 1st trimester & $23(1.6 \%)$ & $162(15 \%)$ & \\
\hline 2nd trimester & $1337(92.9 \%)$ & $435(40.4 \%)$ & $X^{2}=725.08, p<.001$ \\
\hline 3rd trimester & $79(5.5 \%)$ & $481(44.6 \%)$ & \\
\hline
\end{tabular}

Table 1. Demographic characteristics of the samples. $M=$ mean, $S D=$ standard deviation, $t=\mathrm{t}$-test, $X^{2}=$ Chisquare, $p=$ significance level set at $<.05$. Income information was not available in the pre-COVID sample. Education = low: primary education or secondary pre-vocational education, medium: secondary education or vocational education, high: Bachelor or Master's degree or higher (i.e. $\mathrm{PhD}$ ). ${ }^{\star} 11 \%$ of respondents in the COVID sample indicated that they preferred not to answer the question on annual household income.

Support systems. Three support systems were investigated: partner, social network and prenatal healthcare support. Partner and social network support were each assessed through two slider-items from 0.0-10.0, with higher scores indicating more support: "How well were you supported by your partner/social network prior to the COVID-19 outbreak?" for support prior to the pandemic and "How well are you now supported by your partner/social network?" for support during the pandemic. To calculate the partner/social support change, the past reported support was subtracted from the present. As an indication of women's social network composition, we also asked from whom they receive social support. Prenatal healthcare support was assessed with a 3-point Likert scale item "How well are you currently supported by your prenatal healthcare professionals?" with $1=$ very well supported, $2=$ neutral and $3=$ not very well supported. The item was reverse coded in the analyses, such that higher scores consistently indicated better support. Changes in prenatal healthcare support was assessed on a 5-point Likert scale item "Has the support you receive from your primary antenatal healthcare provider(s) changed due to the COVID-19 outbreak?", where: 1 = badly worsened, $2=$ slightly worsened, $3=$ no change, 4 = slightly improved, $5=$ greatly improved. Healthcare support and changes were used as two separate items in the analysis, differently from the composite scores of partner/social support. Moreover, we asked participants who the main prenatal healthcare providers were. The complete instrument can be retrieved online https://osf.io/uqhcv/.

COVID-19-related worries. Worries in relation to COVID-19 were assessed with 8 slider-items from 0.0-10.0, with higher values indicating more worries (Supplementary Table S1). We conducted a factor analysis (FA) and principal component analysis (PCA) to identify the latent factors underlying the data (Supplementary Table S2; Figures S1, S2). Three components were extracted: general COVID-19 worries (worries of contracting symptoms for oneself and others, caring for the family, and overall worries related to the COVID-19 outbreak), social support worries (worries about changes in social and partner support), and work and finances-related (worries about the present and the future work and financial situation). Each component was computed by averaging the items loading on each component, with higher scores indicating more worries.

Statistical analysis. Descriptive statistics summarize the characteristics of the two samples (Table 1). Summary statistics and the correlations amongst the variables are reported in Table 2. For descriptive purposes, we conducted paired sample t-tests assessed perceived support changes during the COVID-19 crisis. Moreover, we also performed repeated measures ANOVA to test mean differences between COVID-19-related worries components: general, social, and work and financial.

To answer the first research question, independent sample t-tests assessed mean differences of anxiety and depression scores between the pre-COVID-19 and COVID-19 samples.

Further, to answer the second research question, namely whether better support reduced COVID-19-related worries, and in turn psychological distress, a parallel mediation analysis was performed separately for each support system. The mediation analyses were conducted with 5000 sample bootstrapping ${ }^{49}$. All analyses were also performed with education and gestational week as control variables, due to statistically significant differences 


\begin{tabular}{|c|c|c|c|c|c|c|c|c|c|c|}
\hline & $M(S D)$ & $\mathbf{N}$ & 1 & 2 & 3 & 4 & 5 & 6 & 7 & 8 \\
\hline 1. General COVID-19 worries & $5.72(2.02)$ & 846 & - & $.524^{\star \star}$ & $.596^{* *}$ & $-.184^{* *}$ & $-.127^{* *}$ & $-.220^{\star *}$ & $.593^{* *}$ & $.551^{* *}$ \\
\hline 2. Social support worries & $2.89(2.41)$ & 1056 & & - & $.425^{* *}$ & $-.319^{* *}$ & $-.358^{* *}$ & $-.152^{\star \star}$ & $.515^{* *}$ & $.500^{* *}$ \\
\hline 3. Work and financial worries & $4.44(2.81)$ & 1025 & & & - & $-.103^{\star *}$ & $-.089^{* *}$ & $-.141^{\star \star}$ & $.439^{* *}$ & $.380^{* *}$ \\
\hline 4. Partner support & $8.76(1.55)$ & 1169 & & & & - & $.249^{* *}$ & $.229^{* *}$ & $-.286^{* *}$ & $-.297^{* *}$ \\
\hline 5. Social support & $7.45(1.72)$ & 1119 & & & & & - & $.132^{* *}$ & $-.231^{* *}$ & $-.213^{* *}$ \\
\hline 6. Healthcare support & $2.53(0.57)$ & 1296 & & & & & & - & $-.213^{\star \star}$ & $-.200^{* *}$ \\
\hline 7. Anxiety symptoms & $41.43(10.61)$ & 1029 & & & & & & & - & $.775^{\star *}$ \\
\hline 8. Depressive symptoms & $6.29(4.87)$ & 1008 & & & & & & & & - \\
\hline
\end{tabular}

Table 2. Summary statistics and correlations among the variables in the study. Notes. $M=$ mean, $S D=$ standard deviation, $N=$ sample, values; ${ }^{\star} p<.05,{ }^{* *} p<.01$.

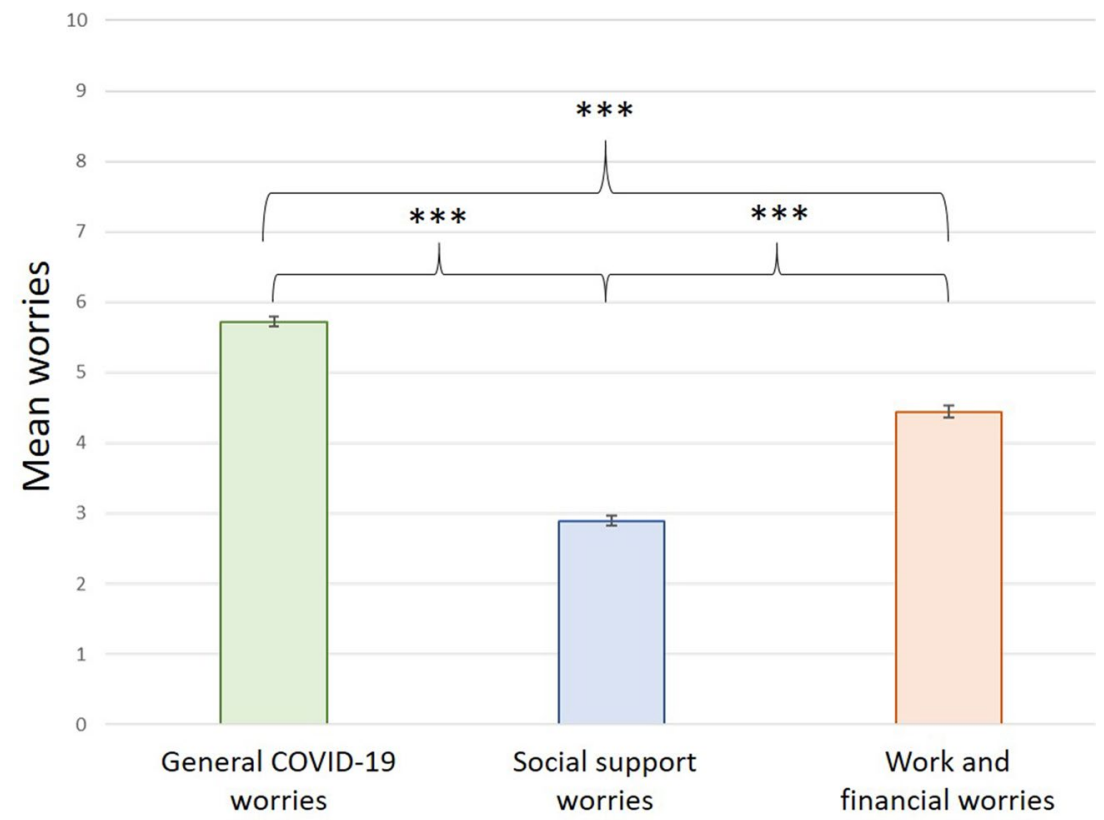

Figure 1. Bar charts depicting the means and standard errors of the COVID-19-related stress: General COVID-19 worries (green), social support worries (blue) and work and financial worries (orange). ${ }^{* *}$ p $<.001$.

between the two samples on these characteristics, but results remained unchanged. The data analyses were conducted with IBM SPSS Statistics $23^{50}$ and PROCESS ${ }^{38}$.

\section{Results}

Preliminary analyses. Support during COVID-19. Paired sample t-tests indicated that women report more $[t(1163)=3.39, p<0.001]$ partner support during the outbreak $[M=8.76, S D=1.54]$ compared to the preCOVID period $[M=8.67, S D=1.48]$. Yet, they reported less $[t(1103)=-7.15, p<0.001]$ social support during the outbreak $[M=7.48, S D=1.71]$ compared to pre-COVID period $[M=7.78, S D=1.58]$. Women reported that they received social support from family $(79.6 \%)$, friends (73.4\%), (mental) healthcare (17.5\%), religious community (3.6\%), and others (colleagues, neighbors, $5 \%$ ). Up to $53 \%$ of pregnant women reported a moderate-strong negative change for prenatal healthcare during the outbreak, $46 \%$ reported no change and $1.3 \%$ reported a positive moderate-strong change. The main prenatal healthcare providers were midwives (83.3\%), gynecologists (29.9\%), general practitioners $(19.2 \%)$, and nurses or doulas (2\%). We were not able to compare healthcare before and after the pandemic, since only one item assessed healthcare changes.

COVID-19-related worries. Results from the repeated measures ANOVA indicated a significant difference between the three reported worries $[F(1,721)=635.72, p<0.001, \eta p 2=0.47]$. Posthoc paired-sample t-tests revealed that general COVID-19 worries were significantly higher than social support worries $[t(780)=34.84$, $p<0.001, d=1.23]$ and work and financial worries $[t(773)=14.67, p<0.001, d=0.49]$. Social support worries were significantly lower than work and financial one $[t(933)=-15.77, p<0.001, d=0.56$; Fig. 1 . 

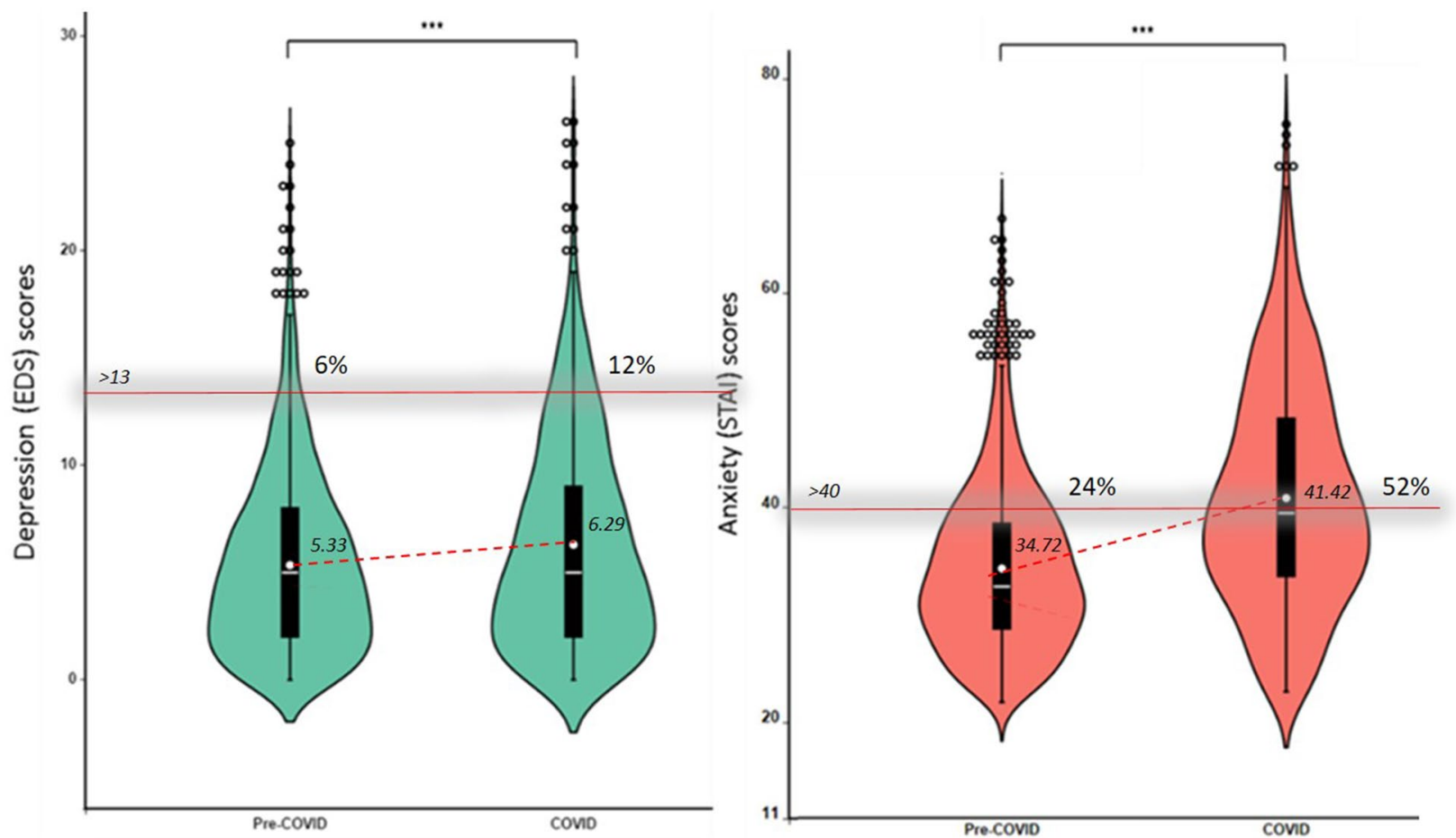

Figure 2. Violin plots illustrating the scores on depression (green) and anxiety (orange) in the pre- and duringCOVID-19 samples. The distribution of the data is represented by the violin shape, with larger width indicating higher value frequency. The mean of each group is represented by the dot, whereas the horizontal bars represent the minimum, the median and the maximum values. The whiskers represent the first and the fifth quantile. The dots represent outlier scores. The percentage of women above the clinical cut-off $>13$ for depression and $>40$ for state anxiety in the Pre-COVID and the COVID sample is noted above the cut-off line. ${ }^{\star * *} \mathrm{p}<.001$.

Pregnant women's mental health before and during COVID-19. Figure 2 illustrates the mean with standard deviation scores for depression and anxiety in the two groups. T-test results revealed significantly higher $[t(2445)=5.18, p<0.001, d=0.21]$ scores for depression in the COVID-19 sample $[M=6.29, S E=0.15$, $N=1008]$, compared to the pre-COVID-19 sample $[M=5.33, S E=0.11, N=1439]$. Likewise, anxiety was significantly higher $[t(2466)=17.98, p<0.001, d=0.71]$ in the COVID-19 sample $[M=41.42, S E=0.33, N=1029]$, compared to the pre-COVID sample $[M=34.72, S E=0.21, N=1439]$. Frequency analyses indicated that $12 \%$ of women experienced clinically relevant depression symptoms during COVID-19 outbreak, compared to $6 \%$ prior to COVID-19 $\left[X^{2}=27.84, p<0.001\right]$. Likewise, clinical anxiety rates were twofold increased in the COVID-19 sample with $52 \%$, compared to the pre-COVID sample with $24 \%\left[X^{2}=214.61, p<0.001\right]$.

Given the variations in anxiety and depression across different trimesters of pregnancy ${ }^{51}$, we assessed differences in symptoms of anxiety and depression between pregnant women in the second trimester of pregnancy, given the prevalence discrepancy of this group in the pre-COVID sample $(n=1337,93 \%)$, compared to the COVID sample $(n=307,40 \%)$. The results on this subgroup confirmed those presented for the whole group, suggesting significantly higher $[t(1642)=1.96, p=0.049, d=0.13]$ scores for depression in the COVID-19 sample $[M=5.91, S E=0.28, N=307]$, compared to the pre-COVID-19 sample [ $M=5.31, S E=0.11, N=1337]$. Likewise for anxiety scores that were significantly higher $[t(1646)=8.71, p<0.001, d=0.59]$ in the COVID-19 sample $[M=40.37, S E=0.61, N=311]$, compared to the pre-COVID sample $[M=34.70, S E=0.21, N=1337]$. Frequency analyses also confirmed a significant twofold increase for depression (10.4\% vs. 5.7\%) and anxiety $(47.9 \%$ vs. $23.9 \%$ ) in the COVID-19 compared to the pre-COVID sample. Note that while women with complicated pregnancies were excluded from the pre-COVID sample, in the COVID-19 sample, all pregnant women were included, and here $14 \%$ reported high-risk pregnancies. We re-ran the t-test and Chi-square analyses excluding high-risk pregnancy women and obtained comparable results. Hence, we retained all women in the COVID-19 sample to maximize the sample size.

Furthermore, due to the high correlation between anxiety and depression in the COVID-19 sample $[r=0.78$, $p<0.001$, we computed a composite score of psychological distress as the mean of centered anxiety and depression variables, to use in further analyses.

Support systems, COVID-19-related worries and psychological distress. Results of the mediation analyses showed that all three support systems (i.e. I. support from one's partner, II. social network and III. healthcare system) individually led to fewer worries (Path a) and less psychological distress (Path c), whereas COVID-19-related worries (M1. COVID-19, M2. support, M3. work and financial) led to an increase in psychological distress (Path b). Moreover, the effect of support on psychological distress was partially mediated by 
a

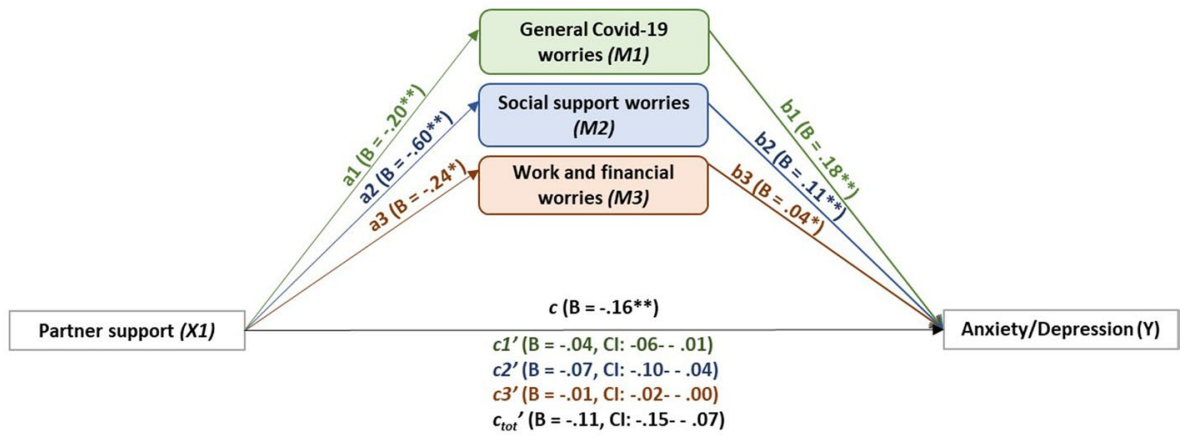

b

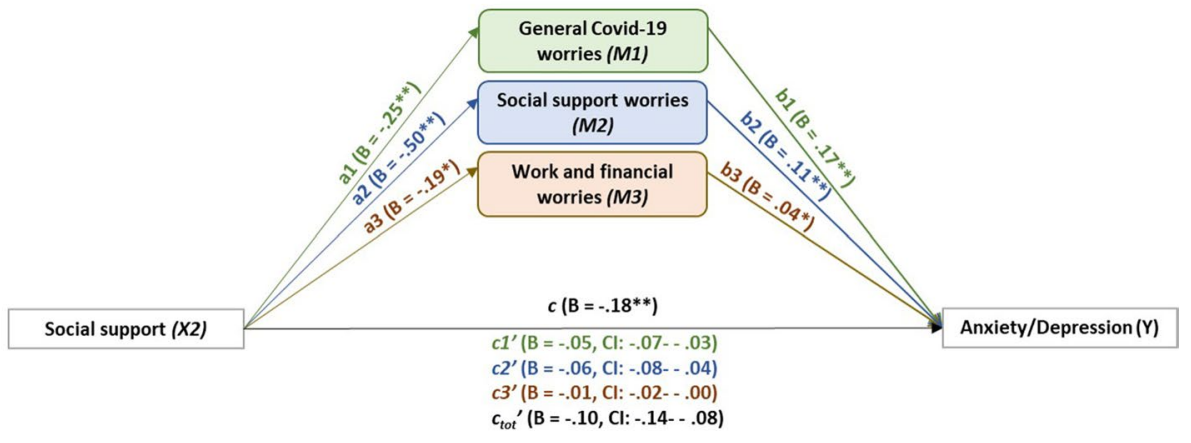

C

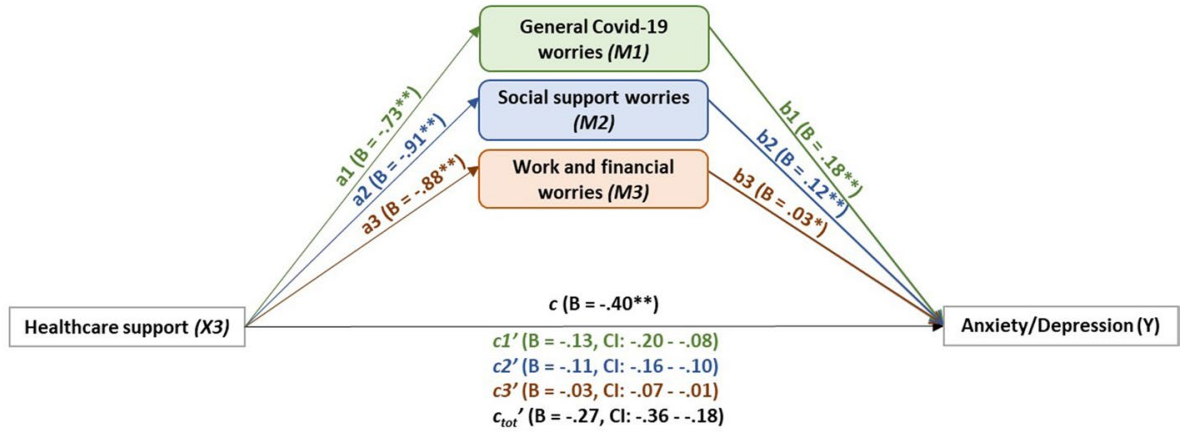

Figure 3. Illustration of the statistical mediation models of the total effect of (a) partner support, (b) social support and (c) healthcare support as IV (X1,2,3) on psychological distress (Y) through the c-path, mediated by general COVID-19 worries (M1, green), social support worries (M2, blue) and work and financial worries (M3, orange), indirect effect shown in c'-paths. The direct effects of the IV on the mediators is shown on the a-paths, and the direct effect of the mediators on the DV is shown in the b-paths. $\mathrm{B}=$ unstandardized coefficient. Significance level was set at $.05{ }^{*} \mathrm{p}<.05,{ }^{* *} \mathrm{p}<.001$.

worries (Path c'), confirming our hypothesis that better support leads to fewer worries, which in turn have a smaller effect on psychological distress. An illustration of our results is provided in Fig. 3, whereas the details of the statistical results of the mediation analyses are reported in Table 3.

\section{Discussion}

Pregnant women reported significantly more symptoms of anxiety and depression (psychological distress) during the COVID-19 crisis, compared to pregnant women prior to the pandemic. More than 1 in 10 women experienced clinically relevant symptoms of depression, and nearly 1 in 2 women experienced clinically relevant symptoms of anxiety. Three types of COVID-19-related worries were identified: general worries about COVID19 , worries about work and finances, and worries about social support, and all led to heightened psychological distress. Note that worries were moderately associated to anxiety symptoms, with correlation coefficients ranging between 0.38 and 0.55 , suggesting that although related, the COVID-19 worries are also distinct from anxiety symptoms. Importantly, having more support (partner, social network, healthcare) was related to fewer worries and consequently less psychological distress. Finally, women reported an increase in partner support during the pandemic, compared to pre-pandemic, but a decrease in social support. More than half of pregnant women also reported a decline in healthcare support.

The results on mental health worsening during pregnancy, at the peak of the Dutch COVID-19 pandemic first lockdown, confirm those from other countries (for a meta-analysis see: ${ }^{21}$ ). Women's most reported worries were general worries about COVID-19 (i.e. worries of contracting symptoms for oneself and others, caring for the family, and overall worries related to the COVID-19 outbreak). This finding is consistent with other reports ${ }^{52}$, 


\begin{tabular}{|c|c|c|c|c|c|c|c|c|c|c|c|}
\hline & & \multicolumn{5}{|c|}{ M1) General COVID-19 worries } & \multicolumn{5}{|c|}{ M2) Social support worries } \\
\hline & & B & SE & $\mathbf{t}$ & $\mathbf{p}$ & CI & B & SE & $t$ & $\mathbf{p}$ & CI \\
\hline \multirow{5}{*}{ I. Partner support $(\mathrm{N}=693)$} & $\mathrm{a}$ & -.20 & .05 & -3.91 & .000 & -.29 to -.10 & -.60 & .06 & -10.82 & .000 & -.71 to -.49 \\
\hline & $\mathrm{b}$ & .18 & .02 & 10.58 & .000 & .15 to. 22 & .11 & .01 & 7.59 & .000 & .08 to. 14 \\
\hline & c & & & & & & & & & & \\
\hline & $\mathrm{c}^{\prime}$ & -.04 & .01 & & & -.06 to -.01 & -.06 & .01 & & & -.10 to -.04 \\
\hline & $c^{\prime}$ tot & & & & & & & & & & \\
\hline \multirow{5}{*}{ II. Social support $(\mathrm{N}=672)$} & $\mathrm{a}$ & -.25 & .05 & -5.51 & .000 & -.34 to -.16 & -.50 & .05 & -9.45 & .000 & -.60 to -.39 \\
\hline & $\mathrm{b}$ & .17 & .02 & 9.83 & .000 & .14 to. 21 & .11 & .01 & 7.60 & .000 & .08 to. 13 \\
\hline & c & & & & & & & & & & \\
\hline & $\mathrm{c}^{\prime}$ & -.04 & .01 & & & -.06 to -.03 & -.05 & .01 & & & -.08 to -.04 \\
\hline & $c^{\prime}$ tot & & & & & & & & & & \\
\hline \multirow{7}{*}{ III. Healthcare support $(\mathrm{N}=694)$} & $\mathrm{a}$ & -.73 & .13 & -5.58 & .000 & $6.77-8.12$ & -.91 & .16 & -5.89 & .000 & -1.22 to -.61 \\
\hline & $\mathrm{b}$ & .18 & .02 & 10.94 & .000 & .14 to. 21 & .12 & .01 & 8.86 & .000 & .09 to. 14 \\
\hline & c & & & & & & & & & & \\
\hline & $c^{\prime}$ & -.13 & .03 & & & -.19 to -.10 & -.11 & .02 & & & -.16 to -.07 \\
\hline & $c^{\prime}$ tot & & & & & & & & & & \\
\hline & & \multicolumn{5}{|c|}{ M3) Work and financial worries } & \multicolumn{5}{|c|}{ DV) Psychological distress } \\
\hline & & B & SE & $t$ & $\mathbf{p}$ & CI & B & SE & $\mathbf{t}$ & $\mathbf{p}$ & CI \\
\hline \multirow{5}{*}{ I. Partner support $(\mathrm{N}=693)$} & $\mathrm{a}$ & -.24 & .07 & -3.43 & .001 & -.37 to -.10 & & & & & \\
\hline & $\mathrm{b}$ & .04 & .01 & 2.99 & .003 & -.09 to -.02 & & & & & \\
\hline & c & & & & & & -.16 & .02 & -7.19 & .000 & -.21 to -.12 \\
\hline & $c^{\prime}$ & -.01 & .00 & & & -.02 to -.00 & & & & & \\
\hline & $c^{\prime}$ tot & & & & & & -.11 & .02 & & & -.16 to -.07 \\
\hline \multirow{5}{*}{ II. Social support $(\mathrm{N}=672)$} & $\mathrm{a}$ & -.19 & .06 & -2.98 & .003 & -.32 to -.07 & & & & & \\
\hline & $\mathrm{b}$ & .04 & .01 & 3.13 & .002 & .01 to.06 & & & & & \\
\hline & c & & & & & & -.19 & .02 & -9.12 & .000 & -.23 to -.05 \\
\hline & $c^{\prime}$ & -.01 & .00 & & & -.02 to -.00 & & & & & \\
\hline & $c^{\prime}$ tot & & & & & & -.10 & .02 & & & -.14 to -.07 \\
\hline \multirow{5}{*}{ III. Healthcare support $(\mathrm{N}=694)$} & $\mathrm{a}$ & -.88 & .18 & -4.84 & .000 & -1.23 to -.52 & & & & & \\
\hline & $\mathrm{b}$ & .03 & .01 & 2.74 & .006 & .01 to. .06 & & & & & \\
\hline & c & & & & & & -.40 & .06 & -2.94 & .000 & -.52 to -.29 \\
\hline & $c^{\prime}$ & -.03 & .01 & & & -.06 to -.01 & & & & & \\
\hline & $c^{\prime}$ tot & & & & & & -.27 & .05 & & & -.37 to -.19 \\
\hline
\end{tabular}

Table 3. Worries as mediators between each source of support and psychological distress (see Fig. 3). The column on the far left presents the predictors (sources of support) for which we ran three separate analyses (I, II, III). The ensuing columns present the mediators $(M 1, M 2, M 3)$, in the order in which they were added to each of the three mediation analyses. If the confidence interval includes 0 , there is no effect. If $c^{\prime}<c$ there is a (partial) mediation effect, if $c^{\prime}$ is no longer significant, then it is a full mediation. $B=$ unstandardized coefficient, $S E=$ standard error of the mean, $t=\mathrm{t}$-test value, $p=\mathrm{p}$ value with significance set at $.05, \mathrm{CI}=$ confidence interval, $\mathrm{N}=$ total sample for each analysis. Path $\mathrm{a}=$ direct effect of each support system on each worry (mediator); Path $b=$ direct effect of each worry on psychological distress; Path $c=$ direct effect of each support system on psychological distress; Path c' $=$ indirect effect of each support system on psychological distress via the suppressing effect of each worry (mediators); Path c'tot = indirect effect of each support system on psychological distress via the suppressing effect of all the worries together (mediators).

and may reflect the little knowledge and information regarding the virus profile in the early stage of the outbreak and its potential vertical transmission to the fetus. Next, worries extended onto the financial domain, probably due to the global recession that the world witnessed with businesses closing, increased unemployment, and governments being challenged to find economic solutions ${ }^{53}$. The least reported worries were worries related to disruptions in social support. This is intriguing, given Dutch COVID-19 containment strategies of social distancing and interpersonal isolation. While all these three worries contribute to psychological distress, general worries about COVID-19 contributed the most to psychological distress, followed by social support worries. Despite women reporting considerable amounts of work and financial worries, these worries had the smallest association with psychological distress. This may be due to the strong COVID-19 financial support measures immediately taken (March 2020) by the Dutch government to protect the economy, which are already known and implemented at the time of our study.

The central finding of this study is that pregnant women reported less worries, and consequently were at lower risk for developing psychological distress, when they experienced more and/or increased support from their partner, social network, and especially their healthcare providers. This is consistent with the buffering hypothesis ${ }^{23}$ 
positing that support helps individuals to deal with hardship. Not surprisingly, however, the profound structural changes in response to the COVID-19 crisis (i.e. family, work, social life, healthcare), might have translated into changes in perceived support. For example, partner support might have increased because of a shift to working at home, which leaves more time for the partner to help in the household and support the pregnant woman. Despite this general increase in partner support, the cases in which it decreased may be associated to relationship conflict, which was shown to increase in non-pregnant populations during COVID-19 ${ }^{54-56}$. Consequently, cases that report low and/or decreased partner support during crises such as COVID-19 deserve further attention, as both indicators were associated with more psychological distress. Contrary to partner support, social network support was lower to that prior to the pandemic. This decline has already been reported in "normal times" during pregnancy and the transition to parenthood, and may be the consequence of life changes associated with this period, including changes in couples' social dynamics ${ }^{57,58}$. A similar trend emerged for healthcare, with 1 in 2 women reporting a decrease in support from their providers during the COVID-19 crisis.

Amongst all sources of support, healthcare provided most relief against COVID-19 worries and heightened psychological distress. Under the circumstances of the sudden and exponential increase in the number of COVID-19 contagions, the healthcare system became saturated, understaffed and often unable to deliver clear and timely information and/or care. Given the primary importance of healthcare providers support for preventing psychological distress, the fact that $53 \%$ of pregnant women reported worsening of their healthcare support is worrying. Additionally, it may be worthwhile for future studies to examine support through objective measures, such as number, nature (in person vs telephone/virtual), and duration of maternity care visits, to gain insights into the structural aspects of the healthcare system that contribute to perceived support and in turn improved mental health. An additional consideration could be the location from which healthcare was received. It could be the case that specific regions and/or hospitals that experienced a higher number of COVD-19 infections may coincide with those in which women reported less perceived support.

Prenatal mental health deterioration during COVID-19 holds important implications for treatment, in light of recent evidence indicating that even prior to the COVID-19 crisis, only 15\% of women with heightened psychological distress are actually treated, possibly due to symptoms going undetected or low treatment uptake rates $^{8,59}$. This phenomenon could have been even exacerbated during the COVID-19 crisis, with even more pregnant women experiencing psychological distress, and even more symptoms going undetected because of worsening healthcare quality. Prenatal mental health worsening thus needs timely attention, especially during times of crisis. Our findings highlight the necessity for universal screening for psychological symptoms during routine health check-ups in the perinatal period, and for interventions aimed at increasing healthcare support during the vulnerable period of pregnancy. Interpersonal psychotherapy (IPT), for example, may be a promising intervention as it has been found effective in improving mood and decreasing depressive symptoms in pregnancy and the perinatal period in general ${ }^{60-62}$. Two main aims of IPT are to enhance social support and decrease interpersonal stress during times of transition. Our findings that higher support leads to fewer worries and in turn psychological distress are in line with the IPT framework and as such provide further support for this therapeutic approach. Other therapies that involve social support, such as person-centered approach (PCA)-based and cognitive-behavioral therapy (CBT)-based interventions, may also be effective in reducing psychological distress in pregnancy ${ }^{63}$. Moreover, online support groups for pregnant women, led by healthcare professionals, might help to address the feelings, emotions and concerns that pregnant women experience as a result of the pregnancy and of living in a pandemic or other type of crisis. Also, timely information should be given about the COVID19 health risks for pregnant women and their unborn child. Taking action is crucial to avoid psychological distress becoming chronic and spilling over into the postnatal period, continuing to affect mother and child, as previously documented in prior natural disaster studies ${ }^{64,65}$. Our findings hold implications for both prevention and intervention work, to establish support systems for vulnerable populations and to facilitate timely access to mental health structures, respectively. Indeed, in future work, it would also be important to carefully document pregnant women's access to mental health during a major crisis such as the COVID-19 pandemic.

This study documented the impact of the COVID-19 pandemic on prenatal mental health, with a large sample of 2858 pregnant women: half assessed prior to the pandemic and half during the pandemic. Moreover, the pandemic data were collected real time through online questionnaires at the peak of the first Dutch COVID-19 outbreak and lockdown at a national level. Nonetheless, some limitations are noteworthy. Firstly, the crosssectional nature of the study design limits the ability to identify the direction of associations. Moreover, the sample did not have a balanced prevalence of women in each trimester of pregnancy, which may have yielded differences in psychological distress. This is however unlikely given that performing the analyses solely on the second trimester subgroup confirmed the results of the whole group. Furthermore, our sample was prevalently well educated, in a marital relationship and reported a medium-high annual household income, indicating a selection bias of the participants in this research. It could be argued, however, that these results may be even more pronounced in women with fewer resources that may contribute to higher stress and mental health issues. An additional limitation was the length of the survey (about 30-45 min), and the fact that demographic information was gathered at the end. This resulted in a large amount of missing demographic data, which informed our decision of not including covariates in the analyses. Nonetheless, the sample was sufficiently large to obtain well-powered reliable results. Finally, the two samples were not perfectly matched: the pre-COVID sample was regional ${ }^{8}$, while the COVID sample was national, and the two samples also differed in education and gestation. However, the two samples were very similar in size and were recruited relatively close in time, providing a unique opportunity for comparison.

To conclude, our study indicated a twofold increase in the prevalence rate of women experiencing depression and anxiety symptoms during the COVID-19 crisis. Importantly, support systems, especially from healthcare providers, counteracted the negative effects on COVID-19 related worries and psychological distress. Our results 
indicate a major opportunity for healthcare providers, including midwives and gynecologists, to reduce COVID19 worries and mental health problems in pregnant women by providing additional support.

\section{Data availability}

Anonymous data can be made available upon request.

Received: 14 June 2021; Accepted: 21 September 2021

Published online: 12 October 2021

\section{References}

1. Alhusen, J. L., Ayres, L. \& DePriest, K. Effects of maternal mental health on engagement in favorable health practices during pregnancy. J. Midwifery Womens Health 61(2), 210-216 (2016).

2. Romero-Gonzalez, B. et al. "I am pregnant. Am I different?": Psychopathology, psychological stress and hair cortisol levels among pregnant and non-pregnant women. J. Psychiatric Res. 131, 235-243 (2020).

3. Woods, S. M., Melville, J. L., Guo, Y., Fan, M. Y. \& Gavin, A. Psychosocial stress during pregnancy. Am. J. Obstet. Gynecol. 202(1), 61-e1 (2010).

4. Hoekzema, E. et al. Pregnancy leads to long-lasting changes in human brain structure. Nat. Neurosci. 20(2), 287-96 (2017).

5. Sieber, S., Germann, N., Barbir, A. \& Ehlert, U. Emotional well-being and predictors of birth-anxiety, self-efficacy, and psychosocial adaptation in healthy pregnant women. Acta Obstet. Gynecol. Scand. 85(10), 1200-7 (2006).

6. Brunton, P. J. Effects of maternal exposure to social stress during pregnancy: consequences for mother and offspring. Reproduction 146(5), R175-89 (2013).

7. Hill, B. et al. A systematic mapping review of the associations between pregnancy intentions and health-related lifestyle behaviours or psychological wellbeing. Prev. Med. Rep. 14, 1069 (2019).

8. Browne, P. D., Bossenbroek, R., Kluft, A., van Tetering, E. M. \& de Weerth, C. Prenatal anxiety and depression: Treatment uptake, barriers, and facilitators in midwifery care. J. Women's Health. 1, 1 (2020).

9. Staneva, A., Bogossian, F., Pritchard, M. \& Wittkowski, A. The effects of maternal depression, anxiety, and perceived stress during pregnancy on preterm birth: A systematic review. Women Birth. 8(3), 179-93 (2015).

10. Van den Bergh, B. R. et al. Prenatal developmental origins of behavior and mental health: The influence of maternal stress in pregnancy. Neurosci. Biobehav. Rev. 1, 1 (2017).

11. Beijers, R., Jansen, J., Riksen-Walraven, M. \& de Weerth, C. Maternal prenatal anxiety and stress predict infant illnesses and health complaints. Pediatrics 126(2), e401-9 (2010).

12. Beijers, R., Buitelaar, J. K. \& de Weerth, C. Mechanisms underlying the effects of prenatal psychosocial stress on child outcomes: beyond the HPA axis. Eur. Child Adolesc. Psychiatry 23(10), 943-56 (2014).

13. Matas-Blanco, C. \& Caparros-Gonzalez, R. A. Influence of Maternal Stress during Pregnancy on Child's Neurodevelopment. Psych. 2(4), 186-97 (2020).

14. World Health Organization. Mental health and psychosocial considerations during the COVID-19 outbreak, 18 March 2020. World Health Organization; 2020.

15. Barzilay, R. et al. Resilience, COVID-19-related stress, anxiety and depression during the pandemic in a large population enriched for healthcare providers. Transl. Psychiatry 10(1), 1-8 (2020).

16. Brooks, S. K. et al. The psychological impact of quarantine and how to reduce it: rapid review of the evidence. The Lancet. 395(10227), 912-20 (2020).

17. Lee, S. A., Jobe, M. C., Mathis, A. A. \& Gibbons, J. A. Incremental validity of coronaphobia: Coronavirus anxiety explains depression, generalized anxiety, and death anxiety. J. Anxiety Disord. 74, 102268 (2020).

18. Caparros-Gonzalez, R. A. \& Alderdice, F. The COVID-19 pandemic and perinatal mental health. J. Reprod. Infant Psychol. 38(3), 223-5 (2020).

19. Caparros-Gonzalez, R. A., Ganho-Ávila, A. \& Torre-Luque, A. D. The COVID-19 pandemic can impact perinatal mental health and the health of the offspring. Behav. Sci. 10(11), 162 (2020).

20. Wastnedge, E. A. et al. Pregnancy and COVID-19. Physiol. Rev. 101(1), 303-18 (2021).

21. Zhang, J., Yu, H., Gao, Y., Xu, Q., Yin, Y., \& Zhou, R. Prevalence of anxiety and depression among pregnant women during the COVID-19 pandemic: a systematic review and meta-analysis.

22. Sommerlad, A., Marston, L., Huntley, J., Livingston, G., Lewis, G., Steptoe, A., \& Fancourt, D. Social relationships and depression during the COVID-19 lockdown: longitudinal analysis of the COVID-19 social study. medRxiv (2020).

23. Cohen, S., \& McKay, G. Social support, stress and the buffering hypothesis: A theoretical analysis. In Handbook of Psychology and Health (Volume IV) 253-267 (Routledge, 2020).

24. Lipsitz, J. D. \& Markowitz, J. C. Mechanisms of change in interpersonal therapy (IPT). Clin. Psychol. Rev. 33(8), 1134-1147 (2013).

25. Cuijpers, P. et al. Interpersonal psychotherapy for depression: a meta-analysis. Am. J. Psychiatry 168(6), 581-592 (2011).

26. Perez, A., Panagiotopoulou, E., Curtis, P. \& Roberts, R. Barriers and facilitators to mood and confidence in pregnancy and early parenthood during COVID-19 in the UK: mixed-methods synthesis survey. BJPsych Open. 7(4), 1 (2021).

27. Rini, C., Schetter, C. D., Hobel, C. J., Glynn, L. M. \& Sandman, C. A. Effective social support: Antecedents and consequences of partner support during pregnancy. Pers. Relat. 13(2), 207-29 (2006).

28. Nyman, T. et al. Perceived social support moderates neural reactivity to emotionally valenced stimuli during pregnancy. Psychophysiology 57(11), e13647 (2020).

29. McCauley, M., Brown, A., Ofosu, B. \& Van Den Broek, N. "I just wish it becomes part of routine care": healthcare providers' knowledge, attitudes and perceptions of screening for maternal mental health during and after pregnancy: a qualitative study. BMC Psychiatry 19(1), 1-8 (2019).

30. Stapleton, L. R. et al. Perceived partner support in pregnancy predicts lower maternal and infant distress. J. Fam. Psychol. 26(3), 453 (2012).

31. Tissera, H., Auger, E., Séguin, L., Kramer, M. S. \& Lydon, J. E. Happy prenatal relationships, healthy postpartum mothers: A prospective study of relationship satisfaction, postpartum stress, and health. Psychol. Health 22, 1-7 (2020).

32. Raycheva, L., Velinova, N., Miteva, N., \& Tomov, M. Impacts of Virtual Communication During Social Isolation of Covid'19. InInternational Conference on Human Systems Engineering and Design: Future Trends and Applications (pp. 63-68). Springer, Cham (2020).

33. Aktas, S. \& Calik, K. Y. Factors affecting depression during pregnancy and the correlation between social support and pregnancy depression. Iran. Red Crescent Med. J. 17(9), 1 (2015).

34. Cairney, J., Boyle, M., Offord, D. R. \& Racine, Y. Stress, social support and depression in single and married mothers. Soc. Psychiatry Psychiatr. Epidemiol. 38(8), 442-9 (2003). 
35. Karavadra, B., Stockl, A., Prosser-Snelling, E., Simpson, P. \& Morris, E. Women's perceptions of COVID-19 and their healthcare experiences: a qualitative thematic analysis of a national survey of pregnant women in the United Kingdom. BMC Pregnan. Childbirth 20(1), 1-8 (2020).

36. Ceulemans, M., Hompes, T. \& Foulon, V. Mental health status of pregnant and breastfeeding women during the COVID-19 pandemic: A call for action. Int. J. Gynecol. Obstet. 151(1), 146-7 (2020).

37. Lebel, C., MacKinnon, A., Bagshawe, M., Tomfohr-Madsen, L. \& Giesbrecht, G. Elevated depression and anxiety symptoms among pregnant individuals during the COVID-19 pandemic. J. Affect. Disord. 277, 5-13 (2020).

38. Hayes, A. F. Introduction to mediation, moderation, and conditional process analysis: A regression-based approach. (Guilford publications, 2017).

39. Browne, P. D., Bolte, A. C., Besseling-van der Vaart, I., Claassen, E. \& de Weerth, C. Probiotics as a treatment for prenatal maternal anxiety and depression: a double-blind randomized pilot trial. Sci. Rep. 11(1), 1-6 (2021).

40. Spielberger, C. D. Assessment of state and trait anxiety: Conceptual and Methodological Issues. Southern Psychologist. 1985.

41. van der Bij, A. K., de Weerd, S., Cikot, R. J., Steegers, E. A. \& Braspenning, J. C. Validation of the dutch short form of the state scale of the Spielberger State-Trait Anxiety Inventory: considerations for usage in screening outcomes. Public Health Genomics 6(2), 84-7 (2003).

42. Grant, K. A., McMahon, C. \& Austin, M. P. Maternal anxiety during the transition to parenthood: A prospective study. J. Affect. Disord. 108(1-2), 101-11 (2008).

43. Elwood, L. S., Wolitzky-Taylor, K. \& Olatunji, B. O. Measurement of anxious traits: A contemporary review and synthesis. Anxiety Stress Coping 25(6), 647-66 (2012).

44. Cox, J. L., Holden, J. M. \& Sagovsky, R. Detection of postnatal depression: Development of the 10-item Edinburgh Postnatal Depression Scale. Br. J. Psychiatry 150(6), 782-6 (1987).

45. Bergink, V. et al. Validation of the Edinburgh Depression Scale during pregnancy. J. Psychosom. Res. 70(4), 385-9 (2011).

46. Matthey, S., Henshaw, C., Elliott, S. \& Barnett, B. Variability in use of cut-off scores and formats on the Edinburgh Postnatal Depression Scale-implications for clinical and research practice. Arch. Womens Ment. Health 9(6), 309-15 (2006).

47. McBride, H. L., Wiens, R. M., McDonald, M. J., Cox, D. W. \& Chan, E. K. The Edinburgh Postnatal Depression Scale (EPDS): A review of the reported validity evidence. Valid Valid Soc Behav Health Sci 1, 157-74 (2014).

48. Thomason, M. E., Graham, A., \& Vantieghem, M. R. COPE: Coronavirus Perinatal Experiences-Impact Survey (COPE-IS). Retrieved from osf. io/uqhcv. 2020.

49. Wright, D. B., London, K. \& Field, A. P. Using bootstrap estimation and the plug-in principle for clinical psychology data. J. Exp. Psychopathol. 2(2), 252-70 (2011).

50. IBM Corp. Released 2020. IBM SPSS Statistics for Windows, Version 27.0. Armonk, NY: IBM Corp

51. Figueiredo, B. \& Conde, A. Anxiety and depression in women and men from early pregnancy to 3-months postpartum. Arch. Womens Ment. Health 14(3), 247-55 (2011).

52. Ravaldi, C., Wilson, A., Ricca, V., Homer, C. \& Vannacci, A. Pregnant women voice their concerns and birth expectations during the COVID-19 pandemic in Italy. Women Birth 1, 1 (2020).

53. Borio, C. The Covid-19 economic crisis: Dangerously unique. Bus. Econ. 55(4), 181-90 (2020).

54. Agüero, J. M. COVID-19 and the rise of intimate partner violence. World Dev. 137, 1017 (2021).

55. Evans, M. L., Lindauer, M. \& Farrell, M. E. A pandemic within a pandemic-Intimate partner violence during Covid-19. N. Engl. J. Med. 383(24), 2302-4 (2020).

56. Moreira, D. N. \& da Costa, M. P. The impact of the Covid-19 pandemic in the precipitation of intimate partner violence. Int. J. Law. Psychiat. 71, 1006 (2020).

57. Redfearn, D. P. et al. Esophageal temperature monitoring during radiofrequency ablation of atrial fibrillation. J. Cardiovasc. Electrophysiol. 6, 589-93 (2005).

58. Hetherington, E., McDonald, S., Williamson, T. \& Tough, S. Trajectories of social support in pregnancy and early postpartum: findings from the All Our Families cohort. Soc. Psychiatry Psychiatr. Epidemiol. 55(2), 259-67 (2020).

59. Goodman, J. H. \& Tyer-Viola, L. Detection, treatment, and referral of perinatal depression and anxiety by obstetrical providers. J. Womens Health 19(3), 477-90 (2020).

60. Spinelli, M. G. \& Endicott, J. Controlled clinical trial of interpersonal psychotherapy versus parenting education program for depressed pregnant women. Am. J. Psychiatry 160(3), 555-62 (2003).

61. Bright, K. S. et al. Interpersonal psychotherapy to reduce psychological distress in perinatal women: A systematic review. Int. J. Environ. Res. Public Health. 17(22), 8421 (2020).

62. Sockol, L. E. A systematic review and meta-analysis of interpersonal psychotherapy for perinatal women. J. Affect. Disord. 232, 316-328 (2018).

63. Branquinho, M. et al. Effectiveness of psychological interventions in the treatment of perinatal depression: A systematic review of systematic reviews and meta-analyses. J. Affect. Disord. 291, 294-306 (2021).

64. King, S., Dancause, K., Turcotte-Tremblay, A. M., Veru, F. \& Laplante, D. P. Using natural disasters to study the effects of prenatal maternal stress on child health and development. Birth Defects Res. C. Embryo Today 96(4), 273-88 (2012).

65. Ping, E. Y. et al. Disaster-related prenatal maternal stress predicts HPA reactivity and psychopathology in adolescent offspring: Project Ice Storm. Psychoneuroendocrinology 1, 1097 (2020).

\section{Acknowledgements}

This research was supported by two Netherlands Organization for Scientific Research VENI grants (Veni. VI.191G.025-to van den Heuvel, 016.195.197-to Beijers), and VICI grant (016.Vici.185.038-to de Weerth), an Early Career Award of the Royal Netherlands Academy of Arts and Sciences (to Beijers), and Sara van Dam Project Grant of the Royal Netherlands Academy of Arts and Sciences (to van den Heuvel), a Jacobs Foundation Advanced Research Fellowship (to de Weerth), two Netherlands Organization for Scientific Research Grants Corona: Fast-track data (to Beijers; to de Weerth), and research funding from the Herbert Simon Research Institute, Tilburg University (to van den Heuvel). The authors also thank the participants who generously shared their time, and Loes van den Heuvel and Annefleur Veens for their help with data collection.

\section{Author contributions}

S.V. performed research, analyses, interpreted the data, wrote the manuscript, R.B. designed and performed research, interpreted the data, wrote the manuscript. P.D.B. performed research, M.C. performed research, provided feedback on the manuscript, H.v.B. performed research, provided feedback on the manuscript, M.v.d.H. designed \& performed research, interpreted the data, wrote the manuscript. C.d.W. designed research, interpreted the data, wrote the manuscript. All authors contributed and approved the final manuscript as submitted. 


\section{Competing interests}

The authors declare no competing interests.

\section{Additional information}

Supplementary Information The online version contains supplementary material available at https://doi.org/ 10.1038/s41598-021-99662-6.

Correspondence and requests for materials should be addressed to S.V.

Reprints and permissions information is available at www.nature.com/reprints.

Publisher's note Springer Nature remains neutral with regard to jurisdictional claims in published maps and institutional affiliations.

(c) (1) Open Access This article is licensed under a Creative Commons Attribution 4.0 International License, which permits use, sharing, adaptation, distribution and reproduction in any medium or format, as long as you give appropriate credit to the original author(s) and the source, provide a link to the Creative Commons licence, and indicate if changes were made. The images or other third party material in this article are included in the article's Creative Commons licence, unless indicated otherwise in a credit line to the material. If material is not included in the article's Creative Commons licence and your intended use is not permitted by statutory regulation or exceeds the permitted use, you will need to obtain permission directly from the copyright holder. To view a copy of this licence, visit http://creativecommons.org/licenses/by/4.0/.

(C) The Author(s) 2021 